Musées, Patrimoine et Culture scientifiques et techniques

148 | 2013

juillet-août 2013

\title{
Une visite scolaire particulière : la grotte
}

Séverine Derolez et Françoise Khantine-Langlois

URL : http://journals.openedition.org/ocim/1248

DOI : $10.4000 /$ ocim. 1248

ISSN : 2108-646X

Éditeur

OCIM

Édition imprimée

Date de publication : 10 juillet 2013

Pagination : 32-38

ISSN : 0994-1908

Référence électronique

Séverine Derolez et Françoise Khantine-Langlois, « Une visite scolaire particulière : la grotte », La Lettre de l'OCIM [En ligne], 148|2013, mis en ligne le 01 juillet 2015, consulté le 30 avril 2019. URL : http:// journals.openedition.org/ocim/1248; DOI : 10.4000/ocim.1248 


\section{Une visite scolaire particulière : la grotte}

\section{Séverine Derolez et Françoise Khantine-Langlois *}

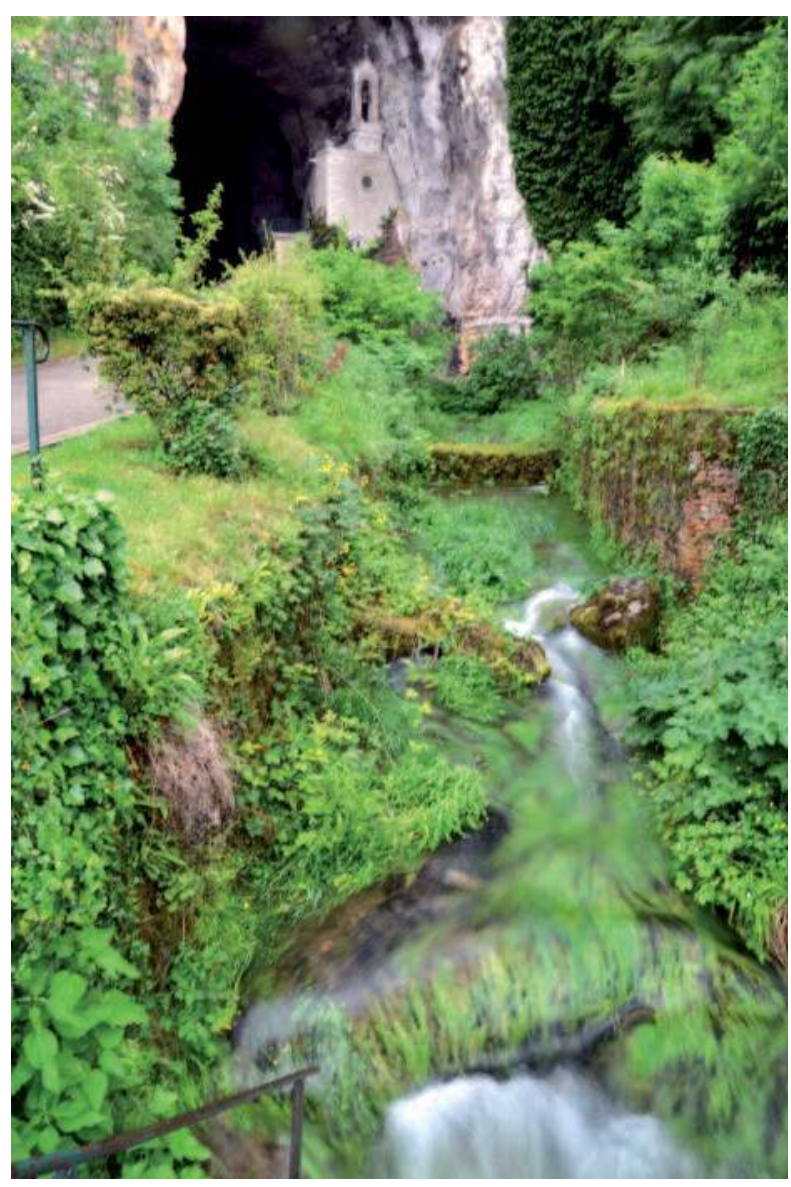

L'entrée des grottes de la Balme, la chapelle et la rivière (c) Julie Olivier

* Séverine Derolez est doctorante à l'université Lyon 1, laboratoire S2HEP severine.derolez@gmail.com Françoise Khantine-Langlois est PRAG Docteur (HDR), IUTA (département Génie chimique, université Claude Bernard Lyon 1, Laboratoire Sciences et Société, Historicité, Éducation et Pratiques f.khantine-langlois@univ.lyonl.fr
Après avoir rappelé les différents textes référents dans le domaine du patrimoine géologique, l'auteur - prenant comme exemple le site de La Balme dans le Rhône - met en lumière les spécificités de la visite scolaire dans une grotte, espace muséal à part entière, en relevant les contraintes du parcours, les adaptations nécessaires et le développement d'outils pédagogiques particuliers.

Pour organiser des sorties géologiques en accord avec les programmes de Sciences de la Vie et de la Terre (SVT), certains enseignants de collèges profitent de la proximité d'une grotte, comme celles de La Balme dans la région Rhône-Alpes. Sur le plan administratif ces visites sont gérées comme les autres visites scolaires. La situation est pourtant assez différente et nous a amenés à nous demander dans quelle mesure les grottes peuvent ou non être assimilées à un espace muséal et quels sont les problèmes spécifiques liés à leur découverte par le public scolaire. Afin de voir comment se préparent et se déroulent, de manière effective, les sorties de classe dans les grottes, un questionnaire a été diffusé auprès des enseignants de collèges (SVT et physique-chimie) et auprès des guides d'un site karstique (année 2010). Ce travail a fait l'objet d'un mémoire de Master 2 (Histoire, Philosophie et Didactique des sciences). Les résultats des questionnaires ont été complétés par des entretiens oraux, dans les établissements et sur le site de visite.

\section{Le statut muséal des grottes}

Se définir en tant que musée

Comme on peut le percevoir à travers l'évolution de la définition du mot «musée » donnée par l'International 
Council of Museums (ICOM), les grottes touristiques sont un peu les laissées pour compte des sites de diffusion scientifique et culturelle. En 1951, le mot musée désigne " tout établissement permanent, administré dans l'intérêt général en vue de conserver, étudier, mettre en valeur par des moyens divers et essentiellement exposer pour la délectation et l'éducation du public un ensemble d'éléments de valeur culturelle: collections d'objets artistiques, historiques scientifiques et techniques, jardins botaniques et zoologiques, aquariums ». Les parcs naturels rejoindront cette définition en 1961, grâce à l'insertion des sites proposant des visites réglementées du public. Dès 1974, le musée est considéré comme étant non plus un établissement mais une institution permanente, sans but lucratif, au service de la société et de son développement, ouverte au public. À partir de 1989 s'adjoindront les centres scientifiques et les planétariums et toute autre institution que le Conseil exécutif considère comme ayant certaines ou toutes les caractéristiques d'un musée, ou donnant à des musées et à des professionnels de musée les moyens de faire des recherches dans les domaines de la muséologie, de l'éducation ou de la formation. Les réserves naturelles seront également incluses dans la famille des musées en 2001. Il faudra attendre 2007 pour noter une évolution dans la définition du «musée » utilisée depuis 1974. Adoptée par la 22e assemblée générale de l'ICOM, à Vienne (Autriche) le 24 août 2007, la définition du «musée » désigne désormais toute institution permanente sans but lucratif, au service de la société et de son développement, ouverte au public, qui acquiert, conserve, étudie, expose et transmet le patrimoine matériel et immatériel de l'humanité et de son environnement à des fins d'études, d'éducation et de délectation. Au fur et à mesure de la précision de la définition, sont intégrés des institutions de plus en plus variées et spécifiques. L'ICOM a pris soin de nommer précisément les nouveaux adhérents à chaque modification : jardins botaniques et zoologiques, parcs naturels, planétarium, réserves naturelles... sans jamais mentionner explicitement le cas des grottes, pourtant déjà très fréquentées à cette époque. Notons que sous le terme de « réserves naturelles » figurent bien des grottes, mais très peu (6 en France) et avec des conditions d'admission exigeantes .

Vers une patrimonialisation des sites naturels À l'échelle internationale, l'un des traités les plus importants en matière de protection du patrimoine est la convention du patrimoine mondial, adoptée par l'UNESCO en 1972 et déjà ratifiée par 190 États au $1^{\text {er }}$ septembre 2012. Bien qu'à l'origine, elle ne soit seulement destinée qu'au patrimoine culturel, son action fut rapidement étendue au patrimoine naturel. Les missions sont claires : protéger ces sites contre les atteintes du temps ou de l'activité des sociétés humaines, les valoriser en y développant un tourisme de qualité afin de répondre à une demande croissante puisque l'économie touristique est appelée à jouer un rôle majeur au XXIe siècle. En novembre 2012, le répertoire du patrimoine mondial comporte 962 sites dont 745 biens culturels, 188 biens naturels et 29 biens mixtes naturels/culturels. Parmi ceux-ci 76 relèvent du patrimoine géologique, soit seulement $11 \%(0,3 \%$ de grottes). Les concrétions des grottes françaises, en tant que témoins exceptionnels du fonctionnement du karst et archives de paléoclimats, ont été proposées en 2006 à l'inscription. Le dossier regroupait 18 cavités dont les Grottes de Choranche situées dans les Alpes (Isère). Le comité n'accéda pas à cette demande. À ce jour aucun bien naturel français ne figure donc au patrimoine mondial de l'UNESCO.

Néanmoins depuis quelques années, de nombreuses initiatives voient le jour afin de mieux faire connaître le patrimoine géologique français. La création de la Conférence Permanente du Patrimoine Géologique a été un levier institutionnel important. Elle est à l'origine de la démarche d'inventaire entreprise par le ministère de l'Environnement et du Développement durable et confiée au Muséum national d'Histoire naturelle. Les programmes scolaires intégrant la géologie dès le plus jeune âge et tout au long de la scolarité, nécessitent l'adaptation de sites de terrain pour y accueillir dans une perspective pédagogique ce public captif. Ainsi, de nombreux espaces protégés ont

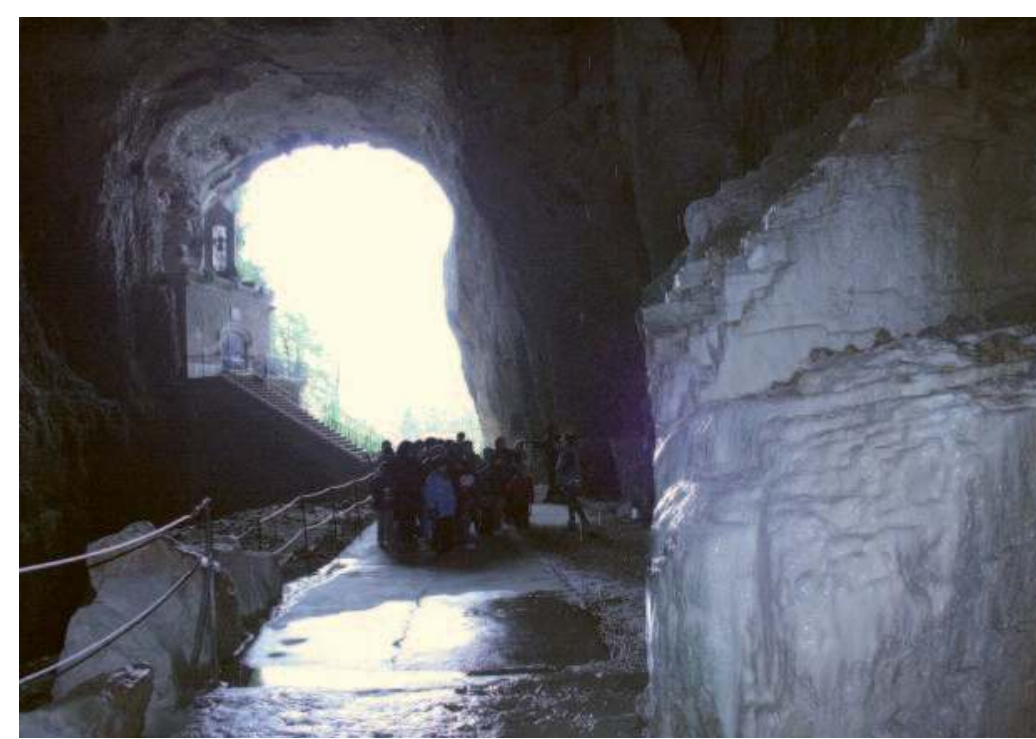

L'accueil des groupes scolaires dans le porche d'entrée est accompagné du bruit du torrent en contrebas. (C) Séverine Derolez 
développé des outils pédagogiques mais aussi de sensibilisation sur le thème de la géologie, assurant ainsi une meilleure diffusion de cette discipline auprès du plus grand nombre.

\section{Les visites scolaires de la classe de $5 \mathrm{e}$ dans I'enseignement scientifique}

\section{Le caractère obligatoire}

J'étudierai ici le cas des sorties scolaires obligatoires, qui s'inscrivent dans le cadre des programmes officiels d'enseignement et qui ont lieu pendant les horaires prévus à l'emploi du temps des élèves ; et notamment celles de la classe de $5 \mathrm{e}$ (Bulletin officiel spécial $\mathrm{n}^{\circ} 6 \mathrm{du}$ 28 août 2008). C'est le programme de physique-chimie qui explicite le mieux l'intérêt de ces visites de façon précise.

"Les visites de musées et des expositions sont également l'occasion de donner aux élèves le goût des sciences, l'envie d'apprendre et de marquer le souvenir. Elles peuvent également avoir un effet déclencheur, car elles constituent une véritable « mise en scène » de ce qui est appris en classe: c'est souvent en de telles occasions que le maître "accrochera » un élève, et l'amènera à porter un regard beaucoup plus constructif sur l'enseignement de la physique et de la chimie » (Annexe G accompagnement du programme de physique-chimie Bulletin officiel spécial n6 du 28 août 2008).

La géologie représente $40 \%$ du programme de SVT de cette classe et doit obligatoirement s'appuyer sur un exemple local. Elle permet d'initier l'élève aux méthodes utilisées par le géologue sur le terrain et d'appliquer un raisonnement par analogie par le recours aux phénomènes actuels pour proposer des explications à ceux du passé. "Dans ce cadre, outre les capacités propres à la culture scientifique et technologique, celles des compétences sociales et civiques peuvent également faire l'objet d'apprentissages : raisonner avec logique et rigueur s'impose dans le cadre de telles reconstitutions par exemple afin de rechercher l'information utile, de l'analyser, la trier, l'organiser et la synthétiser ". La récente circulaire sur les sorties et voyages scolaires de l'enseignement du second degré (circulaire n²011-117 du 3-8-2011) met en avant les nombreux bénéfices retirés par les élèves de ces expériences éducatives et pédagogiques. Cette sortie offre également l'opportunité de construire un projet interdisciplinaire et de mettre en lien plusieurs disciplines pour qui les recommandations « d'extraction de la classe » sont aussi fortement recommandées (sciences de la vie et de la terre, physique-chimie, histoire-géographie, arts plastiques...).
Les recommandations officielles d'une sortie de classe

C'est encore le programme de physique-chimie qui développe le mieux les instructions de préparation d'une telle sortie, mais là encore uniquement pour les musées. La visite d'un musée ou d'une exposition doit s'inscrire, pour être pleinement efficace, dans le programme de la classe et doit en respecter la progression. Elle nécessite une visite préalable du professeur, et doit être conduite à un moment précis de l'avancement du programme. Le professeur peut préparer les élèves à cette visite, leur indiquer les objectifs, leur assigner des tâches précises à réaliser pendant la visite, organiser la communication de leurs découvertes grâce à des documents de visite (fiche, questionnaire, compte rendu...) et structurer leurs acquis au retour en classe. L'impact de la visite sur les élèves peut même entrer dans le système d'évaluation instauré par le professeur. Avant la première visite, il est possible de recueillir l'image que les élèves ont des musées à partir d'un questionnaire ; on peut aussi présenter le musée grâce à son site Internet, mais la découverte et la surprise sont a priori préférables.

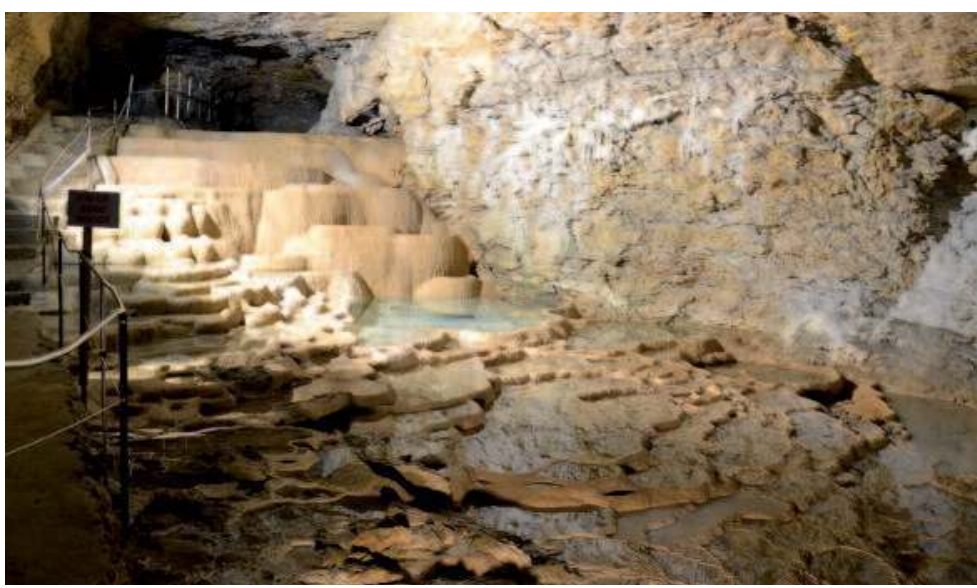

L'amphithéâtre des petits bassins (les gours) avec un passage glissant et bas à proximité de l'eau. (C) Julie Olivier

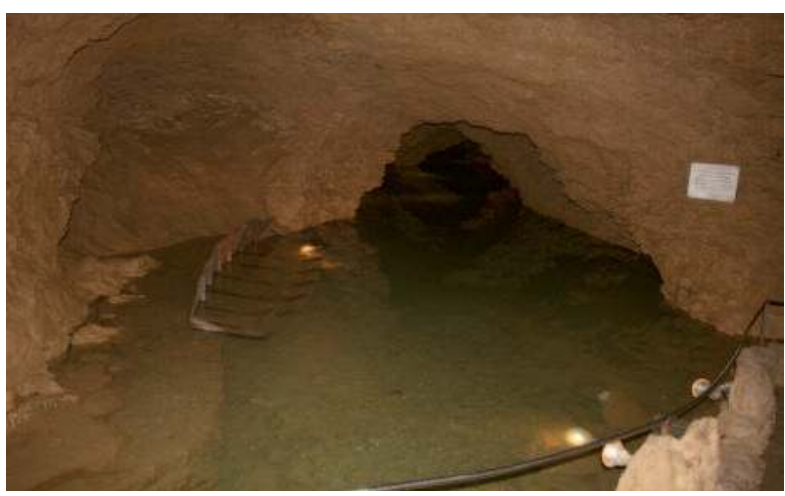

Le lac, galerie inondable ๑) Séverine Derolez 
Pendant la visite, deux démarches peuvent être envisagées : inviter les élèves à compléter un questionnaire à rendre en fin de visite ou leur demander de prendre des notes qui conduiront à des exploitations ultérieures.

En revanche, rien n'évoque le statut des relations entre le professeur, les élèves et le guide au cours de cette sortie. Comme dans la plupart des rapports humains, la relation d'enseignement est sous-tendue par un contrat qui peut être plus ou moins implicite. Il se noue une relation, entre l'enseignant et l'enseigné, qui détermine ce que chaque partenaire a la responsabilité de gérer et dont il est responsable devant l'autre. Il est assez difficile de détailler explicitement ces obligations réciproques, et c'est d'ailleurs plutôt les ruptures de ce contrat qui sont importantes à considérer. Ce contrat s'instaure entre les élèves et l'enseignant, en fonction du milieu dans lequel ils évoluent (Brousseau, 1998). Ce dernier risque d'être mis en défaut par le changement de milieu, et par la présence du guide.

\section{Les spécificités d'une visite scolaire dans une grotte : I'exemple des grottes de La Balme}

\section{La visite des scolaires d'après la municipalité}

Les grottes de la Balme essaient d'attirer le public scolaire en pratiquant un tarif préférentiel, en proposant un discours adapté au programme de géologie et en fournissant un livret pédagogique. Celui-ci se présente sous la forme d'un document numérique de 8 pages comprenant des textes scientifiques, des relevés cartographiques, des schémas, des photographies et deux questionnaires.

Concernant la gestion du groupe, le site présente un texte de recommandations à l'attention des enseignants et accompagnateurs et communiqué via l'affichage à l'entrée (vitres du kiosque). Voici le contenu de ce document, rédigé par le maire de la commune :

"Le guide a pour rôle de conduire les groupes de visiteurs et de présenter le site des grottes de la Balme, il n'a aucune responsabilité en ce qui concerne les enfants; ceux-ci sont placés sous la responsabilité totale et entière des enseignants ou des accompagnateurs. Les personnes encadrant les groupes scolaires doivent donc appliquer et faire appliquer les règles assurant le respect et la sécurité de tous. Les guides et le régisseur se réservent le droit d'exclure des grottes tout individu ou tout groupe nuisant au bon déroulement de la visite et à la sécurité des visiteurs. Les enseignants ou accompagnateurs doivent veiller à ce qu'aucune dégradation ne soit commise. Le Maire».

\section{Préparation effective d'une sortie de classe} Lanalyse des questionnaires distribués aux enseignants et aux guides et les entretiens avec plusieurs d'entre eux font apparaître la dichotomie existant entre les attentes institutionnelles et le "vécu et imposé » par le lieu atypique. En classe, les professeurs préparent souvent ces visites comme ils le font pour une institution muséale, en insistant sur le comportement disciplinaire à adopter à l'extérieur de la classe et expriment leurs attentes vis-à-vis de cette visite (exposé, évaluation orale ou écrite, dossier documentaire...). Le premier point émergant est celui du temps de préparation en classe, qui est en moyenne d'une heure. Certains enseignants font une sensibilisation avant la visite et l'utilisent majoritairement pour un réinvestissement en classe ; mais une part non négligeable d'enseignants ne fait pas de préparation. La visite de la grotte permet alors d'introduire brièvement les parties du programme qui ne seront pas abordées.

Le second point mis en relief et relevé par les guides, est qu'il est fréquent de rencontrer des enseignants qui découvrent le site en même temps que leurs élèves : $52 \%$ contre $48 \%$ l'ayant déjà visité. La plupart du temps, c'est lors d'une visite personnelle que l'enseignant voit l'intérêt qu'il peut en tirer pour son cours et organise par la suite la sortie de classe.

L'étude nous a également montré qu'une majorité des encadrants se procure les documents pédagogiques fournis par le site avant la visite et les utilisent personnellement pour préparer l'excursion. Cependant, les questionnaires ne sont pas transmis en l'état aux élèves. Certains les utilisent pour créer leur propre document qu'ils donneront aux élèves, dans le bus avant d'arriver sur le site, d'autres préféreront demander aux élèves de prévoir un support pour prendre des notes

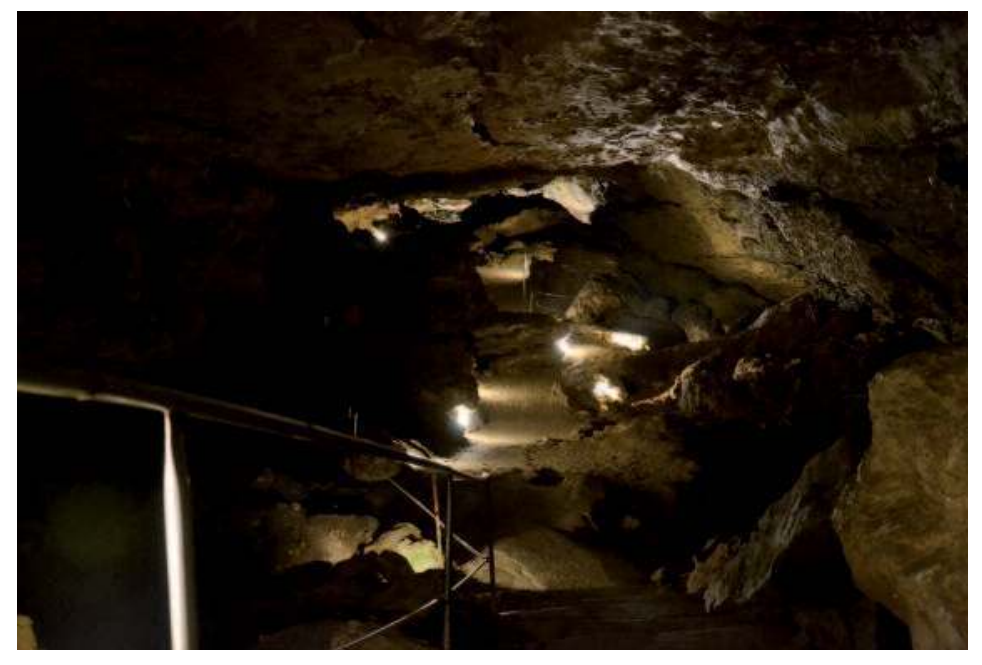

L'escalier sombre et glissant de la galerie du désert. ๑) Julie Olivier 
au cours de la visite. A contrario, seuls $40 \%$ des guides ont déjà consulté les documents pédagogiques, $20 \%$ ne savent pas qu'ils existent, la part restante sait qu'ils existent mais ne les a pas regardés.

Le contenu du livret pédagogique est pourtant partiellement extrait de documents purement disciplinaires, datant d'il y a presque vingt ans (Audra, 1994) peu accessibles aux non spécialistes de la géologie karstique. Les questionnaires ont été réalisés avec ces mêmes documents et donnent lieu à des incohérences et des non-sens frappants. Le manque de démarche interne quant à la conception de ce document nous a amenés à conclure qu'il était difficilement exploitable tel qu'il est proposé actuellement.

\section{L'élève dans un milieu inconnu :}

les singularités du site

En dehors du savoir qu'elle illustre, l'un des effets bénéfiques de la visite d'une grotte réside aussi dans l'impact visuel et émotif du lieu. En cela, son relief est plus proche de la stimulation imaginaire et affective d'une œuvre d'art que d'un objet de savoir scientifique. Le contrat didactique existant in situ est mis en échec par un certain nombre de points, comme la présence d'une nouvelle personne dans la sphère didactique (le guide), le déracinement de l'enseignant de son milieu classique (la classe), la présence d'éléments naturels spontanés (eau, faune), la provocation émotionnelle caractéristique (enfermement, obscurité), parmi d'autres. Dans un lieu comme la grotte, l'action du milieu sur l'élève est prépondérante, et participe directement à l'instauration du nouveau contrat didactique de la classe hors locaux. C'est en général après un certain laps de temps que les collégiens commencent à se sentir à l'aise, s'interrogent sur ce nouvel environnement, et interagissent avec le guide. N'oublions pas que le guide est aussi une personne nouvelle, inconnue des élèves, à laquelle la classe est confrontée, et avec qui elle doit collaborer pour tirer les meilleurs profits de la visite. Le guide doit se faire adopter par cette communauté «classe + professeur » sil veut pouvoir communiquer efficacement. Mais nous l'avons vu à travers l'encart de la mairie, le groupe reste entièrement à la charge de l'enseignant qui en est responsable. Si ce texte couvre juridiquement le site, il est aussi le témoin de l'attente des guides qui se plaignent du manque de présence et de soutien du professeur pendant la visite. Les guides ne souhaitent pas et ne sont pas formés à exercer une autorité sur le groupe.

L'impact du milieu peut aussi se traduire par l'angoisse que peuvent générer certains passages : «je suis trop gros je ne vais pas passer». Cette remarque qui

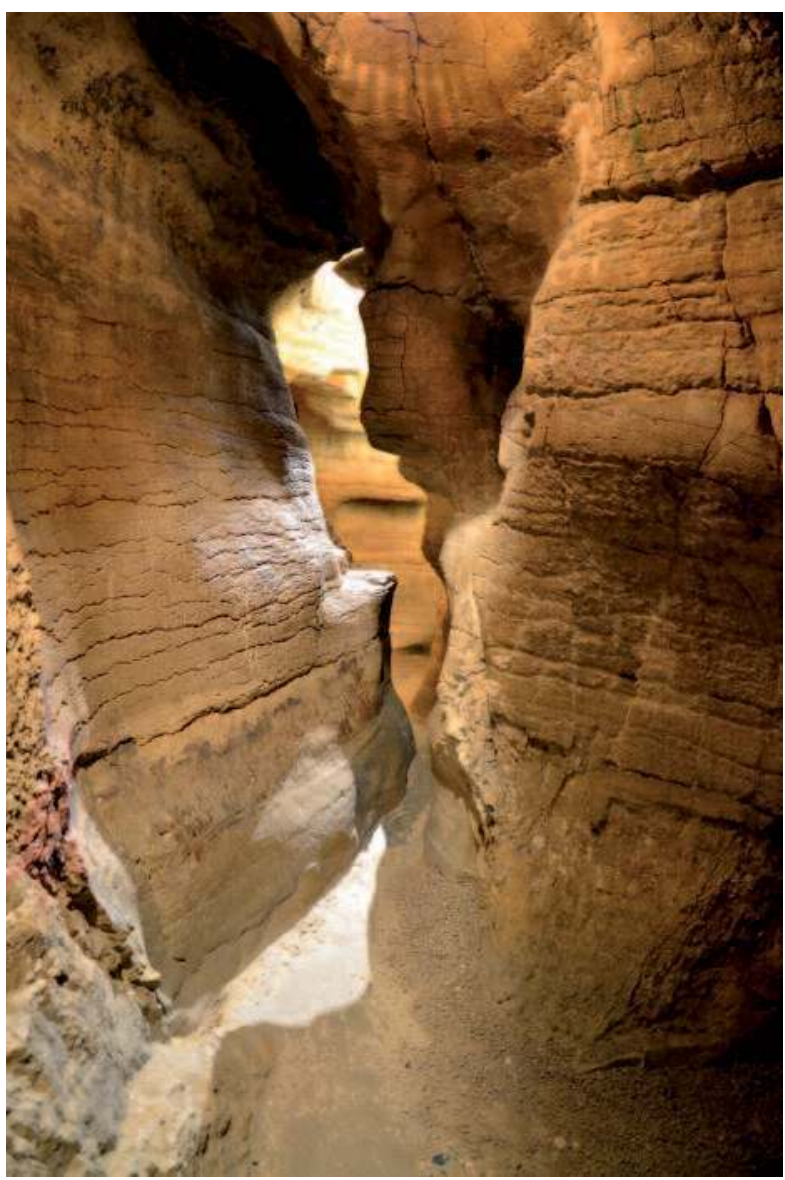

Le labyrinthe de François 1 er avec un passage bas et étroit (๑) Julie Olivier

paraît futile est souvent négligée par les enseignants et banalisée par les guides ; mais cette peur exprimée par les premiers du groupe à l'intention des suivants sous forme de remarque désobligeante de type "le prof ne va jamais passer", "Pierre va rester coincé tellement il est gros", "Laisse ton ventre à l'entrée si tu veux passer » est très mal vécue par les enfants visés, qui se voient relégués en queue de peloton avec inquiétude. Le public adolescent, en dehors de la classe, est une forte entité entre « le monde » et « l'enseignant ", déployant un comportement psychologique qui est une thématique de recherche à part entière : "une des complexités de ce public adolescent repose sur le fait qu'il se trouve dans une période de structuration identitaire, de transition entre l'enfance et l'âge adulte». «En fait, selon les institutions, il faut trowver un contenu qui l'intéresse [l'adolescent] et dans lequel il s'investira. Si sa venue est spontanée, il ne pose pas de problèmes à l'institution car il a des attentes. Mais si la visite est imposée ou imparfaitement préparée (dans le cadre de visites scolaires ou en famille), il devient un public « difficile " (manque d'intérêt d'où une certaine indiscipline ou au contraire une grande passivité )» (Timbart, 2005). 
Dans les grottes de la Balme, après l'étonnement face à la taille du porche d'entrée, la première chose qui marque le visiteur est l'emprise de l'obscurité. Dans un souci de conservation des concrétions, l'éclairage est restreint et non permanent; c'est le guide qui allume et éteint les galeries en fonction de la progression de son groupe. Cet éclairage, installé en fonction de l'itinéraire de la visite, est quasiment imposé par la typologie du lieu. Les spots ne sont présents que pour illuminer les reliefs de la grotte et ne permettent ni la lecture ni l'écriture. Le parcours comportant des petits chemins escarpés et étroits, des salles confinées et exiguës, des balcons à une trentaine de mètres de hauteur, le caractère claustrophobe (peur irrationnelle du noir et de l'obscurité), et achluophobe (peur irrationnelle des espaces confinés, des petites pièces, de l'enfermement) du visiteur peut vite être mis en exergue. La visite comporte également 660 marches à monter et à descendre, et $1 \mathrm{~km}$ de marche à pied, ce qui complique le cheminement pour les enfants à mobilité réduite et l'exclut pour les handicapés moteurs. Il faut faire preuve de vigilance car le parcours passe par des marches sculptées dans le calcaire (glissant), des grilles en acier, des chemins rocheux, des sols recouverts d'argile... La température interne de la grotte est à $14^{\circ} \mathrm{C}$ avec un fort taux d'humidité ; elle ne nécessite pas de précautions particulières en hiver (l'extérieur étant plus frais, le visiteur arrive vêtu chaudement) mais demande de prévoir une petite veste en été. Si la typologie du lieu impose le positionnement de l'éclairage, c'est aussi elle qui prescrit le parcours de la visite et présente des difficultés quant à l'adaptation de la logique explicative en fonction du cheminement temporel de la visite. Il y a un certain nombre d'aller et retour dans le parcours, car le lieu ne permet pas un cheminement linéaire du groupe. De la même manière le relief impose des éléments à voir et donc à commenter, en dehors de toute logique ou démarche d'observation. Si un premier type de calcaire est visible à l'entrée, il faudra attendre l'étape suivante pour en voir d'autres et les comparer avec difficultés. L'une des principales caractéristiques réside dans le fait que le concepteur n'a pas d'objets à exposer, à déplacer, à organiser.

Pour toutes ces raisons, il paraît maintenant évident que l'utilisation d'un questionnaire ou d'une prise de notes lors de la visite est vaine. D'un point de vue pratique, il est impossible de lire dans la grotte, donc d'écrire et de se relire également. On ne peut pas non plus s'asseoir pour dessiner un schéma (sauf à un endroit de la visite). D'un point de vue technique, le papier dans la grotte devient vite fragile (humidité ambiante et gouttes d'eau). D'un point de vue sécuritaire, il n'est pas possible d'avoir les mains occupées, donc de se déplacer avec un cahier, une planche d'écriture ou un bloc notes. Une prise de notes peut aussi s'avérer être une source de danger pour l'élève.

\section{Conclusion}

Une des observations de cette étude montre que de manière assez systématique, les enseignants de SVT, bien que sensibilisés à l'étude de terrain, ne se sont pas posés de questions en rapport avec la spécificité du lieu de visite. Si la visite de cette grotte est une richesse avérée pour les enseignants, elle diffère en plusieurs points d'une visite dans un musée classique. Il ne semble pourtant pas envisageable de pénétrer dans ce lieu sans avertissement quant aux conditions exceptionnelles, aux contraintes spécifiques du lieu.

Le livret pédagogique est le seul moyen d'informer en amont les professeurs sur les conditions exceptionnelles d'une telle visite, les recommandations efficaces à transmettre aux élèves et ce qui est réalisable ou non pendant la visite. Son existence est d'autant plus justifiée pour les organisateurs qui ne connaissent pas le site. Au-delà d'un contenu scientifique adapté et d'un guide d'utilisation du livret lui-même, il serait nécessaire de faire apparaître en détail les conseils pratiques et relationnels. Cela incitera le professeur à prévenir ses élèves des conditions de visites : il fait froid, le sol est glissant, ils resteront debout, il fait relativement sombre, il ne faut rien avoir dans les mains pendant la visite. Bien sûr, il pourra profiter de l'avantage

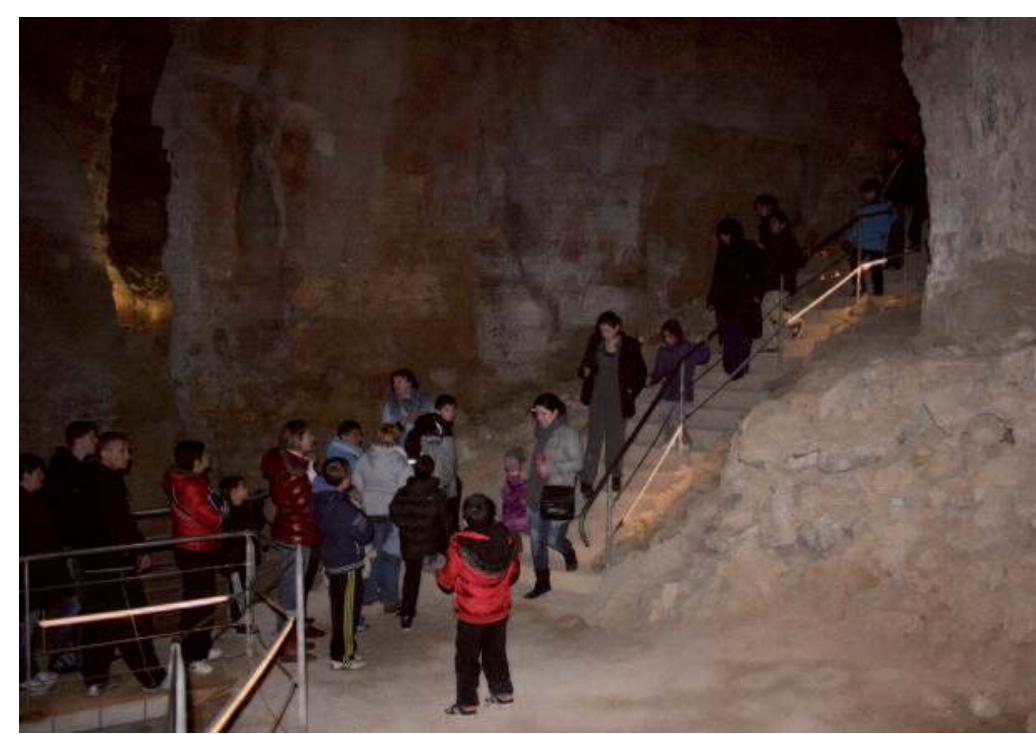

La sortie de la galerie de Mandrin par un escalier étroit (c) Séverine Derolez 
d'une coupure totale du réseau téléphonique, mais devra tout de même recommander d'éviter des chaussures à talons, le port d'un sac à dos trop volumineux et la prise de précautions sanitaires car il n'y a pas de toilettes dans les grottes.

Nous avons donc vu que les différences entre un musée et la grotte sont nombreuses même si en pratique, on les confond encore trop souvent. Les grottes sont un objet muséal difficile à qualifier et à cerner par rapport aux autres lieux connus. Les contraintes qu'elles imposent sont majeures et remettent en question un certain nombre d'études sur l'évolution d'un parcours de visite, le cheminement du visiteur, l'interaction avec l'objet et sa conservation. Mais en sachant prendre en compte ses spécificités, comment ne pas voir l'intérêt majeur que représente ce site ? C'est une expérience à la fois scientifique et sociale, collective, mais qui en apprend également beaucoup sur le comportement individuel de l'élève. En complète adéquation avec le programme scolaire et les attentes de l'Éducation nationale, il est encore dommage de voir qu'une telle visite ne puisse porter tous ses fruits.

\section{Bibliographie}

Audra, P. Karst alpins, quelques exemples régionaux, Karstologia mémoires, n5, 1994, p. 100.

Bakalowicz, M. Connaissance et gestion des ressources en eaux souterraines dans les régions karstiques. Guide technique, n³, SDAGE RhôneMéditerranée-Corse, 1999.

Bompis-Dartout, A. La mise en musée in situ du patrimoine naturel dans le domaine des sciences de la terre. Analyse muséologique d'un musée de site: l'espace Pierres Folles à Saint-Jean-des-Vignes.Thèse pour l'obtention du doctorat, université Claude Bernard, Lyon, 1995.

Brousseau, G. Théorie des situations didactiques. Grenoble : La pensée sauvage, 1998

Cart, G. Les conservateurs de musée et le personnel enseignant, Muséum International, vol. 6, issue IV, UNESCO, 1953, pp. 228-231.

Cayla, N. Le patrimoine géologique de l'arc alpin, de la médiation scientifique à la valorisation géotouristique. Thèse de doctorat de Sciences de la Terre, de l’Univers et de l'Environnement, université de Savoie 2009.

Cone, G., Sanchez, E. et Triquet, E. « Hydroflip » : Un jeu d'enquête et d'orientation sur les eaux souterraines d'un plateau calcaire, Grand N, $n^{\circ} 84,2009$, pp. 9-32.
Derolez, S. Une articulation entre la classe et un site touristique naturel ; comment élaborer un outil pédagogique pertinent pour les deux partenaires ; le cas des grottes de la Balme. Mémoire de M2R, sous la direction de Khantine-Langlois, F. 2010, université Lyon 1.

Eldin, I. Dossier de presse des grottes de la Balme. La Balme Les Grottes, mai 2009.

Emond, A-M. L'éducation muséale vue du Canada, des États-Unis et d'Europe : recherche sur les programmes et les expositions. Québec : Éditions multimondes, 2006.

Girault, Y. Le musée de science : d'un parti pris épistémologique à la prise en compte des publics, Girault, Y. (dir.), L'accueil des publics scolaires dans les muséums, aquariums, jardins botaniques, parcs zoologiques. Paris : L'Harmattan, 2003, pp. 15-50.

Guichard, J. et Martinand, J.-L. Médiatique des sciences. Paris : Éducation et Formation, technologies de l'éducation et de la formation, Presses Universitaires de France (PUF), 2000.

Timbart, N. L'accueil des adolescents dans les institutions muséales scientifiques, La Lettre de l'OCIM, n97, 2005, pp. 24-32.

Triquet, E. Analyse de la genèse d'une exposition de science, pour une approche de la transposition médiatique. Thèse pour l'obtention du doctorat, Lyon, 1993, université Lyon 1.

Triquet, E. et Laperrière, M. Étudier les fossiles au musée. La spécificité des objets et du discours muséal comme point d'ancrage des apprentissages, Aster, n²9, 1999, pp. 148-170.

Triquet, E. La relation École-Musée, Grand N, n66, 2000, pp. 93-106.

Zimmermann, J. Réaliser une enquête. Matériel didactique pour les écoles. Confédération Suisse, Neuchâtel, 2007.

\section{Sites Internet consultés}

Conseil International des Musées : http://icom.museum

Portail national des professionnels de l'éducation : http://eduscol.education.fr/cid47460/culture-scientifique-et-technique.html

Les grottes de la Balme : www.grottes.biz (site officiel)

Organisation des nations-unies pour l'éducation la science et la culture : www.unesco.org/new/fr/natural-sciences/environment/earth-sciences/ geoparks/some-questions-about-geoparks/where-are-the-global-geoparks Agence de développement touristique de la France : www.atout-france.fr/ frequentation-sites-touristiques 